\title{
Existence and Global Exponential Stability of Almost Automorphic Solution for Clifford-Valued High-Order Hopfield Neural Networks with Leakage Delays
}

\author{
Bing $\mathrm{Li}^{1}$ and Yongkun $\mathrm{Li}{ }^{2}{ }^{2}$ \\ ${ }^{1}$ School of Mathematics and Computer Science, Yunnan Minzu University, Kunming, Yunnan 650500, China \\ ${ }^{2}$ Department of Mathematics, Yunnan University, Kunming, Yunnan 650091, China \\ Correspondence should be addressed to Yongkun Li; yklie@ynu.edu.cn
}

Received 13 May 2019; Accepted 1 July 2019; Published 14 July 2019

Academic Editor: Dimitri Volchenkov

Copyright (C) 2019 Bing Li and Yongkun Li. This is an open access article distributed under the Creative Commons Attribution License, which permits unrestricted use, distribution, and reproduction in any medium, provided the original work is properly cited.

In this paper, we study the existence and global exponential stability of almost automorphic solutions for Clifford-valued high-order Hopfield neural networks by direct method. That is to say, we do not decompose the systems under consideration into real-valued systems, but we directly study Clifford-valued systems. Our methods and results are new. Finally, an example is given to illustrate our main results.

\section{Introduction}

In recent years, high-order Hopfield neural networks have become the object of intensive analysis by many scholars because of their stronger approximation characteristics, faster convergence speed, larger storage capacity, and higher fault tolerance than low-order Hopfield neural networks. A lot of excellent research results about their dynamic characteristics have been obtained [1-14].

On the one hand, the Clifford algebra was proposed by the British mathematician William K. Clifford [15] in 1878 and is a generalization of the plural, quaternion, and Glassman algebra. Currently, Clifford algebra has been widely used in various fields such as neural computing, computer and robot vision, image and signal processing, and control problems. Studies have shown that Clifford-valued neural networks are superior to commonly used real-valued neural networks $[16,17]$, so they have become an active research field in recent years. However, because the multiplication of Clifford numbers does not satisfy the commutative law, it has brought great difficulties to the research of Cliffordvalued neural networks. Therefore, the current results on the dynamics of Clifford-valued neural networks are still very rare. At present, only a few papers have been published on the dynamics of Clifford-valued neural networks [18-22]. It is worth mentioning that the results of these mentioned papers are obtained by decomposing Clifford-valued neural networks into real-valued networks. Therefore, it is meaningful to study the dynamics of Clifford-valued neural networks by direct method.

On the other hand, it is well known that periodic and almost periodic oscillations are important dynamic behaviors of neural networks. Almost automorphy is an extension of almost periodicity and plays an important role in better understanding of almost periodicity. Therefore, almost automorphic oscillation is more complex than almost periodic oscillation. Considering the interaction between neurons in a neural network is very complex, it is meaningful to study the almost automorphic oscillation of neural networks.

In addition, time delays are inevitable and may affect and change the dynamic behavior of dynamic systems $[23,24]$. Therefore, neural networks with various delays have been extensively studied. In particular, since K. Gopalsamy [25] first studied the stability of neural networks with leakage delays, a lot of research has been done on neural networks with leakage delays $[6,11,12,26]$. However, there is no research on the Clifford-valued neural network with leakage delays. 
Inspired by the above analysis and discussion, the main purpose of this paper is to study the existence and global exponential stability of almost automorphic solutions for Clifford-valued high-order Hopfield neural networks with leakage delays by direct method; that is, we will study the considered Clifford-valued neural networks directly, instead of converting them to real-valued ones. As far as we know, this is the first paper to study the almost automorphic solutions of Clifford-valued high-order Hopfield neural networks with leakage delays. In addition, this is the first paper to study almost automorphic solutions of Clifford neural networks by direct method. So, our methods and results of this paper are new. Besides, our methods proposed in this paper can be used to study the problem of almost automorphic solutions for other types of Clifford-valued neural networks.

This paper is organized as follows. In Section 2, we introduce some basic definitions and lemmas and give a model description. In Section 3, we study the existence of almost automorphic solutions for Clifford-valued high-order Hopfield neural networks with leakage delays. In Section 4, we investigate the global exponential stability of almost automorphic solutions of the neural networks. In Section 5, an example is given to demonstrate the proposed results. Finally, we draw a brief conclusion in Section 6.

\section{Preliminaries and Model Description}

The real Clifford algebra over $\mathbb{R}^{m}$ is defined as

$$
\mathscr{A}=\left\{\sum_{A \subseteq\{1,2, \ldots, m\}} a^{A} e_{A}, a^{A} \in \mathbb{R}\right\},
$$

where $e_{A}=e_{h_{1}} e_{h_{2}} \cdots e_{h_{\nu}}$ with $A=h_{1} h_{2} \cdots h_{v}, 1 \leq h_{1}<$ $h_{2}<\cdots<h_{v} \leq m$. Moreover, $e_{\emptyset}=e_{0}=1$ and $e_{h}, h=1,2, \ldots, m$ are said to be Clifford generators and satisfy $e_{i}^{2}=-1, i=1,2, \ldots, m$, and $e_{i} e_{j}+e_{j} e_{i}=0, i \neq$ $j, i, j=1,2, \ldots, m$. For convenience, we will denote the product of Clifford generators $e_{h 1}, e_{h_{2}}, \ldots, e_{h v}$ as $e_{h_{1} h_{2} \ldots h_{v}}$. Let $\Pi=\{\emptyset, 1,2, \ldots, A, \ldots, 12 \cdots m\}$; then it is easy to see that $\mathscr{A}=\left\{\sum_{A} a^{A} e_{A}, a^{A} \in \mathbb{R}\right\}$, where $\sum_{A}$ is short for $\sum_{A \in \Pi}$ and $\operatorname{dim} \mathscr{A}=2^{m}$.

For $x=\sum_{A} x^{A} e_{A} \in \mathscr{A}$, we define $\|x\|_{\mathscr{A}}=\max _{A \in \Pi}\left\{\left|x^{A}\right|\right\}$ and for $x=\left(x_{1}, x_{2}, \ldots, x_{n}\right)^{T} \in \mathscr{A}^{n}$, we define $\|x\|_{\mathscr{A}^{n}}=$ $\max _{1 \leq p \leq n}\left\{\left\|x_{p}\right\|_{\mathscr{A}}\right\}$.

The derivative of $x(t)=\sum_{A} x^{A}(t) e_{A}$ is given by $\dot{x}(t)=$ $\sum_{A} \dot{x}^{A}(t) e_{A}$. For more knowledge about Clifford algebra, we refer the reader to [27].

In this paper, we are concerned with the following Clifford-valued high-order Hopfield neural network with delays in the leakage term:

$$
\begin{gathered}
\dot{x}_{p}(t)=-a_{p}(t) x_{p}\left(t-\eta_{p}(t)\right)+\sum_{q=1}^{n} b_{p q}(t) f_{q}\left(x_{q}(t)\right) \\
+\sum_{q=1}^{n} \sum_{l=1}^{n} c_{p q l}(t) g_{q}\left(x_{q}\left(t-\sigma_{p q l}(t)\right)\right) \\
\cdot g_{l}\left(x_{l}\left(t-v_{p q l}(t)\right)\right)+I_{p}(t), \quad p=1,2, \ldots, n,
\end{gathered}
$$

where $x_{p}(t) \in \mathscr{A}$ corresponds to the state of the $p$ th unit at time $t, a_{p}(t) \in \mathbb{R}^{+}$represents the rate with which the $p$ th unit will reset its potential to the resting state when disconnected from the network and external inputs at time $t, b_{p q}(t) \epsilon$ $\mathscr{A}$ denotes the strength of $q$ th unit on $p$ th unit at time $t$, $c_{p q l}(t) \in \mathscr{A}$ is the second-order synaptic weight of the neural networks, $f_{q}(t), g_{q}(t) \in \mathscr{A}$ denote the activation functions, $I_{p}(t) \in \mathscr{A}$ is the external input on the $p$ th at time $t$, and $\eta_{p}(t), \sigma_{p q l}(t), v_{p q l}(t) \in \mathbb{R}^{+}$denote the transmission delays.

Remark 1. When $m$, the number of the generators of $\mathscr{A}$, equals 0,1 , and 2, system (2) degenerates into real-valued, complex-valued, and quaternion-valued systems, respectively.

We will adopt the following notation:

$$
\begin{aligned}
a_{p}^{-} & =\inf _{t \in \mathbb{R}} a_{p}(t), \\
b_{p q}^{+} & =\sup _{t \in \mathbb{R}}\left\|b_{p q}(t)\right\|_{\mathscr{A}}, \\
c_{p q l}^{+} & =\sup _{t \in \mathbb{R}}\left\|c_{p q l}(t)\right\|_{\mathscr{A}}, \\
\eta_{p}^{+} & =\sup _{t \in \mathbb{R}} \eta_{p}(t), \\
\sigma_{p q l}^{+} & =\sup _{t \in \mathbb{R}} \sigma_{p q l}(t), \\
v_{p q l}^{+} & =\sup _{t \in \mathbb{R}} \sigma_{p q l}(t), \\
\xi & =\max _{1 \leq p, q, l \leq n}\left\{\eta_{p}^{+}, \sigma_{p q l}^{+}, v_{p q l}^{+}\right\} .
\end{aligned}
$$

The initial value of system (2) is given by

$$
\begin{aligned}
& x_{p}(s)=\varphi_{p}(s) \in \mathscr{A}, \\
& \dot{x}_{p}(s)=\dot{\varphi}_{p}(s) \in \mathscr{A}, \\
& s s \in[-\xi, 0],
\end{aligned}
$$

where $\varphi_{p} \in C^{1}([-\xi, 0], \mathscr{A})$.

Denote by $U C(\mathbb{R}, \mathbb{R})$ the set of all uniformly continuous functions from $\mathbb{R}$ to $\mathbb{R}$. Let $B C\left(\mathbb{R}, \mathscr{A}^{n}\right)$ denote the set of all bounded continuous functions from $\mathbb{R}$ to $\mathscr{A}^{n}$. Then $\left(B C\left(\mathbb{R}, \mathscr{A}^{n}\right),\|\cdot\|_{0}\right)$ is a Banach space with the norm $\|f\|_{0}=$ $\max _{1 \leq p \leq n} \sup _{t \in \mathbb{R}}\left\{\left\|f_{p}(t)\right\|_{\mathscr{A}}\right\}$, where $f=\left(f_{1}, f_{2}, \ldots, f_{p}\right)^{T} \in$ $B C\left(\mathbb{R}, \mathscr{A}^{n}\right)$.

Definition 2. A function $f \in B C\left(\mathbb{R}, \mathscr{A}^{n}\right)$ is said to be almost automorphic, if for every sequence of real numbers $\left(s_{n}^{\prime}\right)_{n \in \mathbb{N}}$ there exists a subsequence $\left(s_{n}\right)_{n \in \mathbb{N}}$ such that

$$
g(t):=\lim _{n \longrightarrow \infty} f\left(t+s_{n}\right)
$$

is well defined for each $t \in \mathbb{R}$, and

$$
\lim _{n \rightarrow \infty} g\left(t-s_{n}\right)=f(t)
$$

for each $t \in \mathbb{R}$. 
From the above definition, similar to the proofs of the corresponding results in [28], it is not difficult to prove the following two lemmas.

Lemma 3. If $\alpha \in \mathbb{R}, f, g \in A A\left(\mathbb{R}, \mathscr{A}^{n}\right)$, then $\alpha f, f+g, f g \in$ $A A\left(\mathbb{R}, \mathscr{A}^{n}\right)$.

Lemma 4. Let $f \in C\left(\mathscr{A}, \mathscr{A}^{n}\right)$ satisfy the Lipschitz condition and $\varphi \in A A(\mathbb{R}, \mathscr{A})$; then $f(\varphi(\cdot)) \in A A\left(\mathbb{R}, \mathscr{A}^{n}\right)$.

Lemma 5. If $x \in C^{1}(\mathbb{R}, \mathscr{A}), x, x^{\prime} \in A A(\mathbb{R}, \mathscr{A}), \eta \in$ $A A(\mathbb{R}, \mathbb{R}) \cap U C(\mathbb{R}, \mathbb{R})$, then $x(\cdot-\eta(\cdot)) \in A A(\mathbb{R}, \mathscr{A})$.

Proof. Since $x^{\prime} \in A A(\mathbb{R}, \mathscr{A})$ and $\eta \in U C(\mathbb{R}, \mathbb{R}), x(t-\eta(t))$ is uniformly continuous for $t \in \mathbb{R}$. Hence, for each $\varepsilon>0$, there exists a positive number $\delta=\varepsilon / 2$ such that $\left|t_{1}-t_{2}\right|<\delta$ implies

$$
\left\|x\left(t_{1}\right)-x\left(t_{2}\right)\right\|_{\mathscr{A}}<\varepsilon
$$

Since $x, \eta \in A A(\mathbb{R}, \mathscr{A})$, for every sequence of real numbers $\left(s_{n}^{\prime}\right)_{n \in \mathbb{N}}$ there exists a subsequence $\left(s_{n}\right)_{n \in \mathbb{N}}$ such that

$$
\begin{aligned}
& \lim _{n \longrightarrow \infty} x\left(t+s_{n}\right):=\bar{x}(t), \\
& \lim _{n \longrightarrow \infty} \bar{x}\left(t-s_{n}\right)=\bar{x}(t), \\
& \lim _{n \longrightarrow \infty} \eta\left(t+s_{n}\right):=\bar{\eta}(t), \\
& \lim _{n \longrightarrow \infty} \bar{\eta}\left(t-s_{n}\right)=\bar{\eta}(t)
\end{aligned}
$$

for every $t \in \mathbb{R}$. Therefore, there exists a positive integer $N$ such that

$$
\begin{aligned}
\lim _{n \longrightarrow \infty}\left\|x\left(t+s_{n}\right)-\bar{x}(t)\right\|_{\mathscr{A}} & <\frac{\varepsilon}{2}, \\
\lim _{n \longrightarrow \infty}\left|\eta\left(t+s_{n}\right)-\bar{\eta}(t)\right| & <\frac{\varepsilon}{2}
\end{aligned}
$$

for $n>N$ and $t \in \mathbb{R}$. Hence, we have

$$
\begin{aligned}
\| x(t & \left.+s_{n}-\eta\left(t+s_{n}\right)\right)-\bar{x}(t-\bar{\eta}(t)) \|_{\mathscr{A}} \\
\leq & \left\|x\left(t+s_{n}-\eta\left(t+s_{n}\right)\right)-x\left(t+s_{n}-\bar{\eta}(t)\right)\right\|_{\mathscr{A}} \\
& +\left\|x\left(t+s_{n}-\bar{\eta}(t)\right)-\bar{x}(t-\bar{\eta}(t))\right\|_{\mathscr{A}}<\frac{\varepsilon}{2}+\frac{\varepsilon}{2}
\end{aligned}
$$

$=\varepsilon$.

Consequently, $\left\{x\left(t+s_{n}-\eta\left(t+s_{n}\right)\right)\right\}$ converges to $\bar{x}(t-\bar{\eta}(t))$ for each $t \in \mathbb{R}$. Similarly, we can obtain that $\left\{\bar{x}_{1}\left(t-s_{n}-\bar{\eta}\left(t-s_{n}\right)\right)\right\}$ converges to $x(t-\eta(t))$ for each $t \in \mathbb{R}$. Therefore, $x(\cdot-\eta(\cdot)) \in$ $A A(\mathbb{R}, \mathscr{A})$. The proof is complete. tions:

Throughout this paper, we make the following assump-

$$
\begin{aligned}
& \left(H_{1}\right) \text { For } p, q, l=1,2, \ldots, n, a_{p}, \eta_{p}, \sigma_{p q l}, v_{p q l} \in \\
& A A\left(\mathbb{R}, \mathbb{R}^{+}\right) \cap U C(\mathbb{R}, \mathbb{R}) \text { and } \min _{1 \leq p \leq n}\left\{\inf _{t \in \mathbb{R}} a_{p}(t)\right\}> \\
& \\
& 0, b_{p q}, c_{p q l}, I_{p} \in \operatorname{AA}(\mathbb{R}, \mathscr{A}) .
\end{aligned}
$$

$\left(H_{2}\right)$ For $q=1,2, \ldots, n, f_{q}, g_{q}, h_{q} \in C(\mathscr{A}, \mathscr{A})$ and there exist positive constants $L_{q}^{f}, L_{q}^{g}, L_{q}^{h}, G_{p}$ such that

$$
\begin{gathered}
\left\|f_{q}(x)-f_{q}(y)\right\|_{\mathscr{A}} \leq L_{q}^{f}\|x-y\|_{\mathscr{A}}, \\
\left\|g_{q}(x)-g_{q}(y)\right\|_{\mathscr{A}} \leq L_{q}^{g}\|x-y\|_{\mathscr{A}}, \\
\left\|g_{p}(x)\right\|_{\mathscr{A}} \leq G_{p} .
\end{gathered}
$$

Moreover, for $q=1,2, \ldots, n, f_{p}(0)=g_{p}(0)=0$.

$\left(H_{3}\right) \max _{1 \leq p \leq n}\left\{\left(1 / a_{p}^{-}\right) M_{p},\left(1+a_{p}^{+} / a_{p}^{-}\right) M_{p}\right\}:=r<1$, where

$$
\begin{array}{r}
M_{p}=a_{p}^{+} \eta_{p}^{+}+\sum_{q=1}^{n} b_{p q}^{+} L_{q}^{f}+\sum_{q=1}^{n} \sum_{l=1}^{n} c_{p q l}^{+}\left(G_{l} L_{q}^{g}+G_{q} L_{l}^{g}\right), \\
p=1,2, \ldots, n .
\end{array}
$$

\section{The Existence of Almost Automorphic Solutions}

In this section, we study the existence of almost automorphic solutions by the contracting mapping principle.

Let

$$
\begin{aligned}
& \mathbb{Y}=\left\{x \in C^{1}\left(\mathbb{R}, \mathscr{A}^{n}\right): x, x^{\prime} \in A A\left(\mathbb{R}, \mathscr{A}^{n}\right)\right\}, \\
& \mathbb{X}=\left\{\varphi=\left(\varphi_{1}, \varphi_{2}, \ldots, \varphi_{n}\right)^{T} \mid \varphi \in \mathbb{Y}, p=1,2, \ldots, n\right\} .
\end{aligned}
$$

For any $\varphi=\left(\varphi_{1}, \varphi_{2}, \ldots, \varphi_{n}\right)^{T} \in \mathbb{X}$, we define the norm of $\varphi$ as $\|\varphi\|_{\mathcal{X}}=\max \left\{\|\varphi\|_{0},\left\|\varphi^{\prime}\right\|_{0}\right\}$, where $\|\varphi\|_{0}=$ $\max _{1 \leq p \leq n}\left\{\sup _{t \in \mathbb{R}}\left\|\varphi_{p}(t)\right\|_{\mathscr{A}}\right\}$; then $\mathbb{X}$ is a Banach space.

$$
\text { Let } \varphi_{0}(t)=\left(\int_{-\infty}^{t} e^{-\int_{s}^{t} a_{1}(u) d u} I_{1}(s) d s\right. \text {, }
$$
$\left.\int_{-\infty}^{t} e^{-\int_{s}^{t} a_{2}(u) d u} I_{2}(s) d s, \ldots, \int_{-\infty}^{t} e^{-\int_{s}^{t} a_{n}(u) d u} I_{n}(s) d s\right)^{T} \quad$ and take a positive number $R>\left\|\varphi_{0}\right\|_{\mathbb{X}}$.

Set

$$
\begin{aligned}
& \mathbb{X}_{0} \\
& =\left\{\varphi=\left(\varphi_{1}, \varphi_{2}, \ldots, \varphi_{n}\right)^{T} \in \mathbb{X}:\left\|\varphi-\varphi_{0}\right\|_{\mathbb{X}} \leq \frac{r R}{1-r}\right\},
\end{aligned}
$$

and then, for every $\varphi \in \mathbb{X}_{0}$, we have $\|\varphi\|_{\mathbb{X}} \leq\left\|\varphi-\varphi_{0}\right\|_{\mathbb{X}}+$ $\left\|\varphi_{0}\right\|_{\mathbb{X}} \leq r R /(1-r)+R=R /(1-r)$.

Theorem 6. Assume that $\left(H_{1}\right)-\left(H_{3}\right)$ hold. Then system (2) has at least one almost automorphic solution in $\mathbb{X}_{0}$.

Proof. Firstly, it is easy to check that if $x=\left(x_{1}, x_{2}, \ldots, x_{n}\right)^{T} \in$ $\mathbb{X}$ is a solution of the integral equation 


$$
\begin{aligned}
& x_{p}(t) \\
& \quad=\int_{-\infty}^{t} e^{-\int_{s}^{t} a_{p}(u) d u}\left[a_{p}(s) \int_{s-\eta_{p}(s)}^{s} \dot{x}_{p}(u) d u+\sum_{q=1}^{n} b_{p q}(s) f_{q}\left(x_{q}(s)\right)+\sum_{q=1}^{n} \sum_{l=1}^{n} c_{p q l}(s)\right. \\
& \left.\cdot g_{q}\left(x_{q}\left(s-\sigma_{p q l}(s)\right)\right) g_{l}\left(x_{l}\left(s-v_{p q l}(s)\right)\right)+I_{p}(s)\right] d s,
\end{aligned}
$$

where $p=1,2, \ldots, n$, then $x$ is also a solution of system (2).

Secondly, we define an operator $T: \mathbb{X} \longrightarrow B C\left(\mathbb{R}, \mathscr{A}^{n}\right)$ by

$$
T \varphi=\left(T_{1} \varphi, T_{2} \varphi, \ldots, T_{n} \varphi\right)^{T},
$$

where $\varphi \in \mathbb{X}, p=1,2, \ldots, n$,

$$
\begin{aligned}
& \left(T_{p} \varphi\right)(t) \\
& =\int_{-\infty}^{t} e^{-\int_{s}^{t} a_{p}(u) d u}\left[a_{p}(s) \int_{s-\eta_{p}(s)}^{s} \dot{\varphi}_{p}(u) d u+\sum_{q=1}^{n} b_{p q}(s) f_{q}\left(\varphi_{q}(s)\right)+\sum_{q=1}^{n} \sum_{l=1}^{n} c_{p q l}(s)\right. \\
& \left.\cdot g_{q}\left(\varphi_{q}\left(s-\sigma_{p q l}(s)\right)\right) g_{l}\left(\varphi_{l}\left(s-v_{p q l}(s)\right)\right)+I_{p}(s)\right] d s .
\end{aligned}
$$

$$
\begin{aligned}
& \Gamma_{p}(s)=a_{p}(s) \int_{s-\eta_{p}(s)}^{s} \dot{\varphi}_{p}(u) d u+\sum_{q=1}^{n} b_{p q}(s) f_{q}\left(\varphi_{q}(s)\right) \\
&+ \sum_{q=1}^{n} \sum_{l=1}^{n} c_{p q l}(s) g_{q}\left(\varphi_{q}\left(s-\sigma_{p q l}(s)\right)\right) \\
& \cdot g_{l}\left(\varphi_{l}\left(s-v_{p q l}(s)\right)\right)+I_{p}(s), \quad p=1,2, \ldots, n .
\end{aligned}
$$

Then by Lemmas $3-5, \Gamma_{p} \in A A(\mathbb{R}, \mathscr{A})$. We will prove that $T \Gamma_{p} \in A A(\mathbb{R}, \mathscr{A})$.

Let $\left(s_{n}^{\prime}\right)_{n \in \mathbb{N}}$ be a sequence of real numbers; since $a_{p} \in$ $A A(\mathbb{R}, \mathbb{R})$ and $\Gamma_{p} \in A A(\mathbb{R}, \mathscr{A})$, we can extract a subsequence $\left(s_{n}\right)_{n \in \mathbb{N}}$ of $\left(s_{n}^{\prime}\right)_{n \in \mathbb{N}}$ such that, for each $t \in \mathbb{R}$,

$$
\begin{aligned}
& \lim _{n \longrightarrow+\infty} a_{p}\left(t+s_{n}\right):=\bar{a}_{p}(t), \\
& \lim _{n \longrightarrow+\infty} \bar{a}_{p}\left(t-s_{n}\right)=a_{p}(t),
\end{aligned}
$$

$$
p=1,2, \ldots, n
$$

and

$$
\begin{aligned}
\lim _{n \rightarrow+\infty} \Gamma_{p}\left(t+s_{n}\right) & :=\bar{\Gamma}_{p}(t), \\
\lim _{n \rightarrow+\infty} \bar{\Gamma}_{p}\left(t-s_{n}\right) & =\Gamma_{p}(t), \\
& p=1,2, \ldots, n .
\end{aligned}
$$

Set

$$
\left(\overline{T_{p} \Gamma_{p}}\right)(t)=\int_{-\infty}^{t} e^{-\int_{s}^{t} \bar{a}_{p}(u) d u} \bar{\Gamma}_{p}(s) d s
$$


and then we have

$$
\begin{aligned}
& \left\|\left(T_{p} \Gamma_{p}\right)\left(t+s_{n}\right)-\left(\overline{T_{p} \Gamma_{p}}\right)(t)\right\|_{\mathscr{A}} \\
& =\| \int_{-\infty}^{t+s_{n}} e^{-\int_{s}^{t+s_{n}} a_{p}(u) d u} \Gamma_{p}(s) d s \\
& -\int_{-\infty}^{t} e^{-\int_{s}^{t} \bar{a}_{p}(u) d u} \bar{\Gamma}_{p}(s) d s \|_{\mathscr{A}} \\
& =\| \int_{-\infty}^{t+s_{n}} e^{-\int_{s-s_{n}}^{t} a_{p}\left(\delta+s_{n}\right) d \delta} \Gamma_{p}(s) d s \\
& -\int_{-\infty}^{t} e^{-\int_{s}^{t} \bar{a}_{p}(u) d u} \bar{\Gamma}_{p}(s) d s \|_{\mathscr{A}} \\
& =\| \int_{-\infty}^{t} e^{-\int_{u}^{t} a_{p}\left(\delta+s_{n}\right) d \delta} \Gamma_{p}\left(u+s_{n}\right) d u \\
& -\int_{-\infty}^{t} e^{-\int_{s}^{t} \bar{a}_{p}(\delta) d \delta} \bar{\Gamma}_{p}(s) d s \|_{\mathscr{A}} \\
& \quad \leq \| \int_{-\infty}^{t} e^{-\int_{u}^{t} a_{p}\left(\delta+s_{n}\right) d \delta} \Gamma_{p}\left(u+s_{n}\right) d u \\
& -\int_{-\infty}^{t} e^{-\int_{u}^{t} a_{p}\left(\delta+s_{n}\right) d \delta} \bar{\Gamma}_{p}(u) d u \|_{\mathscr{A}} \\
& \quad+\| \int_{-\infty}^{t} e^{-\int_{u}^{t} a_{p}\left(\delta+s_{n}\right) d \delta} \bar{\Gamma}_{p}(u) d u \\
& -\int_{-\infty}^{t} e^{-\int_{u}^{t} \bar{a}_{p}(\delta) d \delta} \bar{\Gamma}_{p}(u) d u \|_{\mathscr{A}}
\end{aligned}
$$

$$
\begin{array}{r}
\leq \int_{-\infty}^{t} e^{-\int_{u}^{t} a_{p}\left(\delta+s_{n}\right) d \delta}\left\|\left(\Gamma_{p}^{1}\left(s+s_{n}\right)-\bar{\Gamma}_{p}(s)\right)\right\|_{\mathscr{A}} d s \\
+\left\|\int_{-\infty}^{t}\left(e^{-\int_{u}^{t} a_{p}\left(\delta+s_{n}\right) d \delta}-e^{-\int_{u}^{t} \bar{a}_{p}(\delta) d \delta}\right) \bar{\Gamma}_{p}(s) d s\right\|_{\mathscr{A}}, \\
p=1,2, \ldots, n .
\end{array}
$$

By the Lebesgue dominated convergence theorem, we obtain that $\lim _{n \rightarrow+\infty}\left(T_{p} \Gamma_{p}\right)\left(t+s_{n}\right)=\left(\overline{T_{p} \Gamma_{p}}\right)(t)$ for each $t \in \mathbb{R}, p=$ $1,2, \ldots, n$. Similarly, one can prove that $\lim _{n \rightarrow+\infty}\left(\overline{T_{p} \Gamma_{p}}\right)(t-$ $\left.s_{n}\right)=\left(T_{p} \Gamma_{p}\right)(t)$ for each $t \in \mathbb{R}, p=1,2, \ldots, n$. Hence, $T_{p} \Gamma_{p} \in$ $A A(\mathbb{R}, \mathscr{A}), p=1,2, \ldots, n$.

Noticing that

$$
\begin{array}{r}
\left(T_{p} \varphi\right)^{\prime}(t)=\Gamma_{p}(t)-a_{p}(t) \int_{-\infty}^{t} e^{-\int_{s}^{t} a_{p}(u) d u} \Gamma_{p}(s) d s, \\
p=1,2, \ldots, n,
\end{array}
$$

similar to the above, we can prove that $\left(T_{p} \varphi\right)^{\prime} \in$ $A A(\mathbb{R}, \mathscr{A}), p=1,2, \ldots, n$. Therefore, $T$ maps $\mathbb{X}$ into itself.

Thirdly, we will prove that $T$ is a self-mapping from $\mathbb{X}_{0}$ to $\mathbb{X}_{0}$. In fact, for each $\varphi \in \mathbb{X}_{0}$, we have

$$
\begin{aligned}
& \left\|(T \varphi)-\varphi_{0}\right\|_{0}=\max _{1 \leq p \leq n_{t \in \mathbb{R}}}\left\|\left(T_{p} \varphi\right)(t)-\varphi_{0}(t)\right\|_{\mathscr{A}} \\
& \leq \max _{1 \leq p \leq n_{t \in \mathbb{R}}}\left\{\int _ { - \infty } ^ { t } e ^ { - \int _ { s } ^ { t } a _ { p } ( u ) d u } \left[\left\|a_{p}(s) \int_{s-\eta_{p}(s)}^{s}\right\| \dot{\varphi}_{p}(u) d u\left\|_{\mathscr{A}}+\sum_{q=1}^{n}\right\| b_{p q}(s)\right.\right. \\
& \cdot f_{q}\left(\varphi_{q}(s)\right)\left\|_{\mathscr{A}}+\sum_{q=1}^{n} \sum_{l=1}^{n}\right\| c_{p q l}(s) g_{q}\left(\varphi_{q}\left(s-\sigma_{p q l}(s)\right)\right) \\
& \left.\left.\times g_{l}\left(\varphi_{l}\left(s-v_{p q l}(s)\right)\right) \|_{\mathscr{A}}\right] d s\right\} \\
& \leq \max _{1 \leq p \leq n}\left\{\int _ { t \in \mathbb { R } } ^ { t } \int _ { - \infty } ^ { - \int _ { s } ^ { t } a _ { p } ( u ) d u } \left(a_{p}^{+}\left\|\varphi^{\prime}\right\|_{0}\right.\right. \\
& \left.\left.\cdot \eta_{p}^{+}+\sum_{q=1}^{n} b_{p q}^{+} L_{q}^{f}\|\varphi\|_{0}+\sum_{q=1}^{n} \sum_{l=1}^{n} c_{p q l}^{+} G_{l} L_{q}^{g}\|\varphi\|_{0}\right)\right\} \\
& \quad \leq \max _{1 \leq p \leq n}\left\{\frac{1}{a_{p}^{-}}\left(a_{p}^{+} \eta_{p}^{+}+\sum_{q=1}^{n} b_{p q}^{+} L_{q}^{f}+\sum_{q=1}^{n} \sum_{l=1}^{n} c_{p q l}^{+} G_{l} L_{q}^{g}\right)\right\}\|\varphi\|_{\mathbb{X}} \leq \max _{1 \leq p \leq n}\left\{\frac{1}{a_{p}^{-}} M_{p}\right\}\|\varphi\|_{\mathbb{X}}
\end{aligned}
$$


and

$$
\begin{aligned}
& \left\|\left(\Psi \varphi-\varphi_{0}\right)^{\prime}\right\|_{0} \leq \max _{1 \leq p \leq n}\left\{\| a_{t \in \mathbb{R}}(t) \int_{t-\eta_{p}\left(t, \varphi_{p}(t)\right)}^{t} \dot{\varphi}_{p}(u) d u+\sum_{q=1}^{n} b_{p q}(t) f_{q}\left(\varphi_{q}(t)\right)\right. \\
& \quad+\sum_{q=1}^{n} \sum_{l=1}^{n} c_{p q l}(t) g_{q}\left(\varphi_{q}\left(t-\sigma_{p q l}\left(t, \varphi_{q}(t)\right)\right)\right) g_{l}\left(\varphi_{l}\left(s-v_{p q l}\left(t, \varphi_{l}(t)\right)\right)\right)\left\|_{\mathscr{A}}+\right\| \int_{-\infty}^{t} a_{p}(t) \\
& \left.\quad \cdot e^{-\int_{s}^{t} a_{p}(u) d u}\left[a_{p}(s) \int_{s-\eta_{p}\left(s, \varphi_{p}(s)\right)}^{s} \dot{\varphi}_{p}(u) d u+\sum_{q=1}^{n} b_{p q}(s) f_{q}\left(\varphi_{q}(s)\right)+\sum_{q=1}^{n} \sum_{l=1}^{n} c_{p q l}(s) g_{q}\left(\varphi_{q}\left(s-\sigma_{p q l}\left(s, \varphi_{q}(s)\right)\right)\right) g_{l}\left(\varphi_{l}\left(s-v_{p q l}\left(s, \varphi_{l}(s)\right)\right)\right)\right] d s\|\|_{\mathscr{A}}\right\} \\
& \quad \leq \max _{1 \leq p \leq n}\left\{\left(a_{p}^{+} \eta_{p}^{+}+\sum_{q=1}^{n} b_{p q}^{+} L_{q}^{f}+\sum_{q=1}^{n} \sum_{l=1}^{n} c_{p q l}^{+} G_{l} L_{q}^{g}\right)\|\varphi\|_{\mathbb{X}}+\frac{a_{p}^{+}}{a_{p}^{-}}\left(a_{p}^{+} \eta_{p}^{+}+\sum_{q=1}^{n} b_{p q}^{+} L_{q}^{f}+\sum_{q=1}^{n} \sum_{l=1}^{n} c_{p q l}^{+} G_{l} L_{q}^{g}\right)\|\varphi\|_{\mathbb{X}}\right\} \leq \max _{1 \leq p \leq n}\left\{\left(1+\frac{a_{p}^{+}}{a_{p}^{-}}\right) M_{p}\right\}\|\varphi\|_{\mathbb{X}} .
\end{aligned}
$$

\section{Thus, we obtain}

$$
\begin{aligned}
& \left\|\left(T \varphi-\varphi_{0}\right)\right\|_{X} \\
& =\max _{1 \leq p \leq n}\left\{\sup _{t \in \mathbb{R}}\left\|\left(T \varphi-\varphi_{0}\right)\right\|_{0}, \sup _{t \in \mathbb{R}}\left\|\left(T \varphi-\varphi_{0}\right)^{\prime}\right\|_{0}\right\} \\
& \quad \leq \max _{1 \leq p \leq n}\left\{\frac{1}{a_{p}^{-}} M_{p},\left(1+\frac{a_{p}^{+}}{a_{p}^{-}}\right) M_{p}\right\}\|\varphi\|_{\mathbb{X}}=r\|\varphi\|_{\mathbb{X}} \\
& \quad \leq \frac{r R}{1-r} .
\end{aligned}
$$

Fourthly, we will prove that $T$ is a contracting mapping. In fact, for any $\varphi, \psi \in \mathbb{X}_{0}$, we have that

$$
\begin{aligned}
& \|T \varphi-T \psi\|_{0} \leq \max _{1 \leq p \leq n}\left\{\int _ { - \infty } ^ { t } e ^ { - \int _ { s } ^ { t } a _ { p } ( u ) d u } \left[\left\|a_{p}(s) \int_{s-\eta_{p}(s)}^{s}\left[\dot{\varphi}_{p}(u)-\dot{\psi}_{p}(u)\right] d u\right\|_{\mathscr{A}}+\sum_{q=1}^{n}\left\|b_{p q}(s)\left[f_{q}\left(\varphi_{q}(s)\right)-f_{q}\left(\psi_{q}(s)\right)\right]\right\|_{\mathscr{A}}\right.\right. \\
& \left.\left.\quad+\sum_{q=1}^{n} \sum_{l=1}^{n}\left\|c_{p q l}(s)\left[g_{q}\left(\varphi_{q}\left(s-\sigma_{p q l}(s)\right)\right) g_{l}\left(\varphi_{l}\left(s-v_{p q l}(s)\right)\right)-g_{q}\left(\varphi_{q}\left(s-\sigma_{p q l}(s)\right)\right) g_{l}\left(\varphi_{l}\left(s-v_{p q l}(s)\right)\right)\right]\right\|_{\mathscr{A}}\right] d s\right\} \\
& \quad \leq \max _{1 \leq p \leq n}\left\{\int_{-\infty}^{t} e^{-a_{p}^{-}(t-s)} d s\left[a_{p}^{+} \eta_{p}^{+}\left\|\dot{\varphi}_{q}-\dot{\psi}_{q}\right\|_{0}+\sum_{q=1}^{n} b_{p q}^{+} L_{q}^{f}\left\|\varphi_{q}-\psi_{q}\right\|_{0}+\sum_{q=1}^{n} \sum_{l=1}^{n} c_{p q l}^{+}\left[G_{q} L_{l}^{g}\|\varphi-\psi\|_{0}+G_{l} L_{q}^{g}\|\varphi-\psi\|_{0}\right]\right]\right. \\
& \quad \leq \max _{1 \leq p \leq n} \frac{1}{a_{p}^{-}} M_{p}\|\varphi-\psi\|_{\mathbb{X}} \leq r\|\varphi-\psi\|_{\mathbb{X}}
\end{aligned}
$$

and

$$
\begin{aligned}
\left\|(T \varphi-T \psi)^{\prime}\right\|_{0} & \leq \max _{1 \leq p \leq n}\left(1+\frac{a_{p}^{+}}{a_{p}^{-}}\right) M_{p}\|\varphi-\psi\|_{\mathbb{X}} \\
& \leq r\|\varphi-\psi\|_{\mathbb{X}} .
\end{aligned}
$$

Hence, by $\left(\mathrm{H}_{3}\right), T$ is a contracting mapping principle. Therefore, there exists a unique fixed-point $\varphi^{*} \in \mathbb{X}_{0}$ such that
$T \varphi^{*}=\varphi^{*}$, which implies that system (2) has an almost automorphic solution in $\mathbb{X}_{0}$. The proof is complete.

\section{Global Exponential Stability}

In this section, we investigate the global exponential stability of almost automorphic solutions by reduction to absurdity. 
Definition 7. Let $x=\left(x_{1}, x_{2}, \ldots, x_{n}\right)^{T}$ be an almost automorphic solution of system (2) with the initial value $\varphi=\left(\varphi_{1}, \varphi_{2}, \ldots, \varphi_{n}\right)^{T} \in C\left([-\xi, 0], \mathscr{A}^{n}\right)$ and let $y=$ $\left(y_{1}, y_{2}, \ldots, y_{n}\right)^{T}$ be an arbitrary solution of system (2) with the initial value $\psi=\left(\psi_{1}, \psi_{2}, \ldots, \psi_{n}\right)^{T} \in C\left([-\xi, 0], \mathscr{A}^{n}\right)$, respectively. If there exist positive constants $\lambda$ and $M$ such that

$$
\|x(t)-y(t)\|_{1} \leq M\|\varphi-\psi\|_{\xi} e^{-\lambda t}, \quad \forall t>0,
$$

where

$$
\begin{aligned}
& \|x(t)-y(t)\|_{1}=\max \left\{\|x(t)-y(t)\|_{\mathscr{A}^{n}},\right. \\
& \left.\left\|(x(t)-y(t))^{\prime}\right\|_{\mathscr{A}^{n}}\right\}, \\
& \|\varphi-\psi\|_{\xi}=\max \left\{\sup _{t \in[-\xi, 0]} \max _{1 \leq p \leq n}\left\|\varphi_{p}(t)-\psi_{p}(t)\right\|_{\mathscr{A}},\right. \\
& \left.\sup _{t \in[-\xi, 0]} \max _{1 \leq p \leq n}\left\|\left(\varphi_{p}(t)-\psi_{p}(t)\right)^{\prime}\right\|_{\mathscr{A}}\right\} .
\end{aligned}
$$

Then the almost automorphic solution of system (2) is said to be globally exponentially stable.

Theorem 8. Assume that $\left(H_{1}\right)-\left(H_{3}\right)$ hold. Then system (2) has a unique pseudo almost automorphic solution that is globally exponentially stable.

Proof. By Theorem 6, system (2) has a pseudo almost periodic solution. Let $x(t)$ be an almost automorphic solution of (2) with the initial value $\varphi(t)$ and let $y(t)$ be an arbitrary solution with the initial value $\psi(t)$. Set $z_{p}(t)=x_{p}(t)-y_{p}(t), \phi_{p}(t)=$ $\varphi_{p}(t)-\psi_{p}(t)$, and we have

$$
\begin{gathered}
\dot{z}_{p}(t)=-a_{p}(t) z_{p}\left(t-\eta_{p}(t)\right)+\sum_{q=1}^{n} b_{p q}(t) \tilde{f}_{q}\left(z_{q}(t)\right) \\
+\sum_{q=1}^{n} \sum_{l=1}^{n} c_{p q l}(t) \widetilde{g}_{q}\left(z_{q}\left(t-\sigma_{p q l}(t)\right)\right) \\
\cdot \widetilde{g}_{q}\left(z_{q}\left(t-v_{p q l}(t)\right)\right), \quad p=1,2, \ldots, n,
\end{gathered}
$$

where

$$
\begin{aligned}
& \tilde{f}_{q}\left(z_{q}(t)\right)=f_{q}\left(x_{q}(t)\right)-f_{q}\left(y_{q}(t)\right), \\
& \tilde{g}_{q}\left(z_{q}\left(t-\sigma_{p q l}(t)\right)\right) \tilde{g}_{q}\left(z_{q}\left(t-v_{p q l}(t)\right)\right) \\
& =g_{q}\left(x_{q}\left(t-\sigma_{p q l}(t)\right)\right) g_{q}\left(x_{q}\left(t-v_{p q l}(t)\right)\right) \\
& \quad-g_{q}\left(y_{q}\left(t-\sigma_{p q l}(t)\right)\right) g_{q}\left(y_{q}\left(t-v_{p q l}(t)\right)\right) .
\end{aligned}
$$

Let $\Theta_{p}$ and $\Delta_{p}$ be defined by

$$
\begin{gathered}
\Theta_{p}(\omega)=a_{p}^{-}-\omega-\left(a_{p}^{+} \eta_{p}^{+} e^{\omega \eta_{p}^{+}}+\sum_{q=1}^{n} b_{p q}^{+} L_{q}^{f} e^{\omega}\right. \\
\left.+\sum_{q=1}^{n} \sum_{l=1}^{n} c_{p q l}^{+}\left(L_{q}^{g} e^{\omega \sigma_{p q l}^{+}}+L_{l}^{g} e^{\omega \nu_{p q l}^{+}}\right)\right)
\end{gathered}
$$

and

$$
\begin{aligned}
& \Delta_{p}(\omega)=a_{p}^{-}-\omega-\left(a_{p}^{+}+a_{p}^{-}-\omega\right)\left(a_{p}^{+} \eta_{p}^{+} e^{\omega \eta_{p}^{+}}\right. \\
& \left.+\sum_{q=1}^{n} b_{p q}^{+} L_{q}^{f} e^{\omega}+\sum_{q=1}^{n} \sum_{l=1}^{n} c_{p q l}^{+}\left(L_{q}^{g} e^{\omega \sigma_{p q l}^{+}}+L_{l}^{g} e^{\omega v_{p q l}^{+}}\right)\right),
\end{aligned}
$$

where $p=1,2, \ldots, n$. When $\omega=0$, we get

$$
\Theta_{p}(0)>0
$$

$$
\text { and } \Delta_{p}(0)>0 \text {, }
$$

$$
p=1,2, \ldots, n .
$$

Since $\Theta_{p}(\omega), \Delta_{p}(\omega)$ are continuous on $[0,+\infty)$ and $\Theta_{p}(\omega), \Delta_{p}(\omega) \longrightarrow-\infty$ as $\omega \longrightarrow+\infty$, there exist $\varepsilon_{p}, \varepsilon_{p}^{*}>0$ such that $\Theta_{p}\left(\varepsilon_{p}\right)=\Delta\left(\varepsilon_{p}^{*}\right)=0$ and $\Theta_{p}(\varepsilon)>0$ for $\varepsilon \in\left(0, \varepsilon_{p}\right)$ and $\Delta\left(\varepsilon_{p}^{*}\right)>0$ for $\varepsilon \in\left(0, \varepsilon_{p}^{*}\right), p=1,2, \ldots, n$. Let $\alpha=\min _{1 \leq p \leq n}\left\{\omega_{p}, \varepsilon_{p}^{*}\right\}$; then we have

$$
\begin{aligned}
& \Theta_{p}(\alpha) \geq 0, \\
& \Delta_{p}(\alpha) \geq 0,
\end{aligned}
$$

$$
p=1,2, \ldots, n .
$$

So we can take a positive constant $\lambda \in \epsilon$ $\left(0, \min \left\{\alpha, a_{1}^{-}, a_{2}^{-}, \ldots, a_{n}^{-}\right\}\right)$. Obviously, we have

$$
\begin{aligned}
& \Theta_{p}(\lambda)>0, \\
& \Theta_{p}(\lambda)>0,
\end{aligned}
$$

$$
p=1,2, \ldots, n,
$$

which implies that

$$
\begin{aligned}
& \frac{1}{a_{p}^{-}-\lambda}\left(a_{p}^{+} \eta_{p}^{+} e^{\lambda \eta_{p}^{+}}+\sum_{q=1}^{n} b_{p q}^{+} L_{q}^{f} e^{\lambda}\right. \\
& \left.\quad+\sum_{q=1}^{n} \sum_{l=1}^{n} c_{p q l}^{+}\left(G_{l} L_{q}^{g} e^{\omega \sigma_{p q l}^{+}}+G_{q} L_{l}^{g} e^{\omega v_{p q l}^{+}}\right)\right)<1, \\
& \left(1+\frac{a_{p}^{+}}{a_{p}^{-}-\lambda}\right)\left(a_{p}^{+} \eta_{p}^{+} e^{\lambda \eta_{p}^{+}}+\sum_{q=1}^{n} b_{p q}^{+} L_{q}^{f} e^{\lambda}\right. \\
& \left.\quad+\sum_{q=1}^{n} \sum_{l=1}^{n} c_{p q l}^{+}\left(G_{l} L_{q}^{g} e^{\omega \sigma_{p q l}^{+}}+G_{q} L_{l}^{g} e^{\omega v_{p q l}^{+}}\right)\right)<1,
\end{aligned}
$$

where $p=1,2, \ldots, n$. Let $M=\max _{1 \leq p \leq n}\left\{a_{p}^{-} / M_{p}\right\}$. From $\left(H_{3}\right)$, we have $M>1$. Hence, for $p=1,2, \ldots, n$, we can obtain

$$
\begin{aligned}
\frac{1}{M} & \leq \frac{1}{a_{p}^{-}-\lambda} \times\left(a_{p}^{+} \eta_{p}^{+} e^{\lambda \eta_{p}^{+}}+\sum_{q=1}^{n} b_{p q}^{+} L_{q}^{f} e^{\lambda}\right. \\
& \left.+\sum_{q=1}^{n} \sum_{l=1}^{n} c_{p q l}^{+}\left(G_{l} L_{q}^{g} e^{\omega \sigma_{p q l}^{+}}+G_{q} L_{l}^{g} e^{\omega \nu_{p q l}^{+}}\right)\right) .
\end{aligned}
$$


By (31), we have

$$
\begin{gathered}
\dot{z}_{p}(t)+a_{p}(t) z_{p}(t)=a_{p}(t) \int_{t-\eta_{p}(t)}^{t} \dot{z}_{p}(s) d s \\
+\sum_{q=1}^{n} b_{p q}(t) \tilde{f}_{q}\left(z_{q}(t)\right)+\sum_{q=1}^{n} \sum_{l=1}^{n} c_{p q l}(t) \\
\cdot \widetilde{g}_{q}\left(z_{q}\left(t-\sigma_{p q l}(t)\right)\right) \widetilde{g}_{q}\left(z_{q}\left(t-v_{p q l}(t)\right)\right) .
\end{gathered}
$$

Multiplying (41) by $e^{\int_{0}^{s} a_{p}(u) d u}$ and integrating on $[0, t]$, we have

$$
\begin{gathered}
z_{p}(t)=\phi_{p}(0) e^{-\int_{0}^{t} a_{p}(u) d u} \\
+\int_{0}^{t} e^{-\int_{s}^{t} a_{p}(u) d u}\left[a_{p}(s) \int_{s-\eta_{p}(s)}^{s} \dot{z}_{p}(u) d u+\sum_{q=1}^{n} b_{p q}(s) \widetilde{f}_{q}\left(z_{q}(s)\right)+\sum_{q=1}^{n} \sum_{l=1}^{n} c_{p q l}(s)\right. \\
\left.\cdot \widetilde{g}_{q}\left(z_{q}\left(s-\sigma_{p q l}(s)\right)\right) \tilde{g}_{q}\left(z_{q}\left(s-v_{p q l}(s)\right)\right)\right] d s, \\
p=1,2, \ldots, n .
\end{gathered}
$$

It is easy to see that

$$
\begin{aligned}
& \|z(t)\|_{1}=\|\phi(t)\|_{1} \leq\|\phi\|_{\xi} \leq M\|\phi\|_{\xi} e^{-\lambda t} \\
& t \in(-\xi, 0] \text {. }
\end{aligned}
$$

We claim that

$$
\|z(t)\|_{1} \leq M\|\phi\|_{\xi} e^{-\lambda t}, \quad t \in[0,+\infty)
$$

To prove (44), we show that, for any $\beta>1$, the following inequality holds:

$$
\|z(t)\|_{1} \leq \beta M\|\phi\|_{\xi} e^{-\lambda t}, \quad t>0
$$

If (45) is false, then there must be some $t_{1}>0$ such that

$$
\begin{aligned}
\left\|z\left(t_{1}\right)\right\|_{1} & =\max _{1 \leq p \leq n}\left\{\left\|z_{p}\left(t_{1}\right)\right\|_{\mathscr{A}},\left\|\dot{z}_{p}\left(t_{1}\right)\right\|_{\mathscr{A}}\right\} \\
& =\beta M\|\phi\|_{\xi} e^{-\lambda t_{1}}
\end{aligned}
$$

and

$$
\|z(t)\|_{1} \leq \beta M\|\phi\|_{\xi} e^{-\lambda t}, \quad t \in\left[-\xi, t_{1}\right]
$$

By (39), (42), (46), and (47), we have

$$
\begin{aligned}
& \left\|z_{p}\left(t_{1}\right)\right\|_{\mathscr{A}} \leq\|\phi\|_{\xi} e^{-t_{1} a_{p}^{-}}+\beta M\|\phi\|_{\xi} \\
& \cdot e^{-\lambda s} \int_{0}^{t_{1}} e^{-\left(t_{1}-s\right) a_{p}^{-}}\left[a_{p}^{+} \eta_{p}^{+} e^{\lambda \eta_{p}^{+}}+\sum_{q=1}^{n} b_{p q}^{+} L_{q}^{f}\right.
\end{aligned}
$$

$$
\begin{aligned}
& \left.+\sum_{q=1}^{n} \sum_{l=1}^{n} c_{p q l}^{+}\left(G_{l} L_{q}^{g} e^{\lambda \sigma_{p q l}^{+}}+G_{q} L_{l}^{g} e^{\lambda v_{p q l}^{+}}\right)\right] d s \\
& \leq \beta M\|\phi\|_{\xi} e^{-\lambda t_{1}}\left[\frac{e^{\left(\lambda-a_{p}^{-}\right) t_{1}}}{\beta M}+\frac{1}{a_{p}^{-}-\lambda}\left(a_{p}^{+} \eta_{p}^{+} e^{\lambda \eta_{p}^{+}}\right.\right.
\end{aligned}
$$$$
+\sum_{q=1}^{n} b_{p q}^{+} L_{q}^{f}
$$$$
\left.+\sum_{q=1}^{n} \sum_{l=1}^{n} c_{p q l}^{+}\left(G_{l} L_{q}^{g} e^{\lambda \sigma_{p q l}^{+}}+G_{q} L_{l}^{g} e^{\lambda v_{p q l}^{+}}\right)\right)(1
$$

$$
\left.\left.-e^{\left(\lambda-a_{p}^{-}\right) t_{1}}\right)\right] \leq \beta M\|\phi\|_{\xi} e^{-\lambda t_{1}}\left[e ^ { ( \lambda - a _ { p } ^ { - } ) t _ { 1 } } \left(\frac{1}{M}\right.\right.
$$$$
-\frac{1}{a_{p}^{-}-\lambda} \sum_{q=1}^{n}\left(a_{p}^{+} \eta_{p}^{+} e^{\lambda \eta_{p}^{+}}+\sum_{q=1}^{n} b_{p q}^{+} L_{q}^{f}\right.
$$$$
\left.+\sum_{q=1}^{n} \sum_{l=1}^{n} c_{p q l}^{+}\left(G_{l} L_{q}^{g} e^{\lambda \sigma_{p q l}^{+}}+G_{q} L_{l}^{g} e^{\lambda v_{p q l}^{+}}\right)\right)+\frac{1}{a_{p}^{-}-\lambda}
$$

$$
\cdot \sum_{q=1}^{n}\left(a_{p}^{+} \eta_{p}^{+} e^{\lambda \eta_{p}^{+}}+\sum_{q=1}^{n} b_{p q}^{+} L_{q}^{f}\right.
$$


Complexity

9

$$
\begin{aligned}
& \left.\left.+\sum_{q=1}^{n} \sum_{l=1}^{n} c_{p q l}^{+}\left(G_{l} L_{q}^{g} e^{\lambda \sigma_{p q l}^{+}}+G_{q} L_{l}^{g} e^{\lambda \nu_{p q l}^{+}}\right)\right)\right] \\
& \leq \beta M\|\phi\|_{\xi} e^{-\lambda t_{1}}\left[\frac { 1 } { a _ { p } ^ { - } - \lambda } \sum _ { q = 1 } ^ { n } \left(a_{p}^{+} \eta_{p}^{+} e^{\lambda \eta_{p}^{+}}+\sum_{q=1}^{n} b_{p q}^{+} L_{q}^{f}\right.\right. \\
& \left.\left.+\sum_{q=1}^{n} \sum_{l=1}^{n} c_{p q l}^{+}\left(G_{l} L_{q}^{g} e^{\lambda \sigma_{p q l}^{+}}+G_{q} L_{l}^{g} e^{\lambda \nu_{p q l}^{+}}\right)\right)\right] \\
& <\beta M\|\phi\|_{\xi} e^{-\lambda t_{1}}, \quad p=1,2, \ldots, n .
\end{aligned}
$$

Finding the derivative of (42), then by (39), (46), and (47), we have

(48)

$$
\begin{aligned}
& \left\|\dot{z}_{p}\left(t_{1}\right)\right\|_{\mathscr{L}} \leq a_{p}^{+}\|\phi\|_{\xi} e^{-a_{p} t_{1}}+a_{p}^{+} \eta_{p}^{+} e^{-\lambda\left(t_{1}-\eta_{p}^{+}\right)} \beta M\|\phi\|_{\xi} \\
& +e^{-\lambda t_{1}} \beta M\|\phi\|_{\xi} \sum_{q=1}^{n} b_{p q}^{+} L_{q}^{f} \\
& +\beta M\|\phi\|_{\xi} \sum_{q=1}^{n} \sum_{l=1}^{n} c_{p q l}^{+}\left(G_{l} L_{q}^{g} e^{-\lambda\left(t_{1}-\sigma_{p q l}^{+}\right)}\right. \\
& \left.+G_{q} L_{l}^{g} e^{-\lambda\left(t_{1}-\gamma_{p q l}^{+}\right)}\right) \\
& +\beta M\|\phi\|_{\xi} \int_{0}^{t_{1}} a_{p}^{+} e^{-\left(t_{1}-s\right) a_{p}^{-}}\left[a_{p}^{+} \eta_{p}^{+} e^{-\lambda\left(s-\eta_{p}^{+}\right)}+e^{-\lambda s} \sum_{q=1}^{n} b_{p q}^{+} L_{q}^{f}+\sum_{q=1}^{n} \sum_{l=1}^{n} c_{p q l}^{+}\left(G_{l} L_{q}^{g} e^{-\lambda\left(t_{1}-\sigma_{p q l}^{+}\right)}\right.\right. \\
& \left.\left.+G_{q} L_{l}^{g} \mathrm{e}^{-\lambda\left(t_{1}-v_{p q l}^{+}\right)}\right)\right] d s \\
& \leq \beta M\|\phi\|_{\xi} \\
& \cdot e^{-\lambda t_{1}}\left[\frac{a_{p}^{+} e^{\left(\lambda-a_{p}^{-}\right) t_{1}}}{\beta M}+\frac{a_{p}^{+}}{a_{p}^{-}-\lambda}\left(a_{p}^{+} \eta_{p}^{+} e^{\lambda \eta_{p}^{+}}+\sum_{q=1}^{n} b_{p q}^{+} L_{q}^{f}+\sum_{q=1}^{n} \sum_{l=1}^{n} c_{p q l}^{+}\left(G_{l} L_{q}^{g} e^{\lambda \sigma_{p q l}^{+}}\right.\right.\right. \\
& \left.\left.\left.+G_{q} L_{l}^{g} e^{\lambda v_{p q l}^{+}}\right)\right)\left(1-e^{\left(\lambda-a_{p}^{-}\right) t_{1}}\right)\right] \\
& \leq \beta M\|\phi\|_{\xi} e^{-\lambda t_{1}}\left[a _ { p } ^ { + } e ^ { ( \lambda - a _ { p } ^ { - } ) t _ { 1 } } \left(\frac{1}{M}\right.\right. \\
& -\frac{1}{a_{p}^{-}-\lambda} \sum_{q=1}^{n}\left(a_{p}^{+} \eta_{p}^{+} e^{\lambda \eta_{p}^{+}}+\sum_{q=1}^{n} b_{p q}^{+} L_{q}^{f}+\sum_{q=1}^{n} \sum_{l=1}^{n} c_{p q l}^{+}\left(G_{l} L_{q}^{g} e^{\lambda \sigma_{p q l}^{+}}+G_{q} L_{l}^{g} e^{\lambda v_{p q l}^{+}}\right)\right)+(1 \\
& \left.+\frac{a_{p}^{+}}{a_{p}^{-}-\lambda}\right)\left(a_{p}^{+} \eta_{p}^{+} e^{\lambda \eta_{p}^{+}}+\sum_{q=1}^{n} b_{p q}^{+} L_{q}^{f}+\sum_{q=1}^{n} \sum_{l=1}^{n} c_{p q l}^{+}\left(G_{l} L_{q}^{g} e^{\lambda \sigma_{p q l}^{+}}\right.\right. \\
& \left.\left.\left.+G_{q} L_{l}^{g} e^{\lambda \nu_{p q l}^{+}}\right)\right)\right] \\
& \leq \beta M\|\phi\|_{\xi} e^{-\lambda t_{1}}\left(1+\frac{a_{p}^{+}}{a_{p}^{-}-\lambda}\right)
\end{aligned}
$$




$$
\begin{aligned}
& \cdot\left(a_{p}^{+} \eta_{p}^{+} e^{\lambda \eta_{p}^{+}}+\sum_{q=1}^{n} b_{p q}^{+} L_{q}^{f}+\sum_{q=1}^{n} \sum_{l=1}^{n} c_{p q l}^{+}\left(G_{l} L_{q}^{g} e^{\lambda \sigma_{p q l}^{+}}\right.\right. \\
& \left.\left.+G_{q} L_{l}^{g} e^{\lambda v_{p q l}^{+}}\right)\right)<\beta M\|\phi\|_{\xi} e^{-\lambda t_{1}}, \quad p=1,2, \ldots, n .
\end{aligned}
$$

From (48) and (49), we have $\left\|z\left(t_{1}\right)\right\|_{1}<\beta M\|\phi\|_{\xi} e^{-\lambda t_{1}}$, which contradicts equality (46). Hence, (45) holds. Letting $\beta \longrightarrow 1$, then (44) holds. Therefore, the almost automorphic solution of (2) is globally exponentially stable. The proof is complete.

\section{Example}

In this section, we give an example to show the feasibility of our results obtained in this paper.

Example 1. In system (2), let $m=n=2$, and take

$$
\begin{aligned}
& f_{q}\left(x_{q}\right)=\frac{1}{22} e_{0} \sin x_{q}^{0}+\frac{1}{20} e_{1} \sin x_{q}^{1}+\frac{1}{21} e_{2} \sin x_{q}^{2}+\frac{1}{27} e_{12} \sin x_{q}^{12}, \quad q=1,2, \\
& g_{q}\left(x_{q}\right)=\frac{1}{16} e_{0} \sin x_{q}^{0}+\frac{1}{20} e_{1} \sin x_{q}^{1}+\frac{1}{15} e_{2} \sin x_{q}^{2}+\frac{1}{18} e_{12} \sin x_{q}^{12}, \quad q=1,2, \\
& \left(\begin{array}{l}
a_{1}(t) \\
a_{2}(t)
\end{array}\right)=\left(\begin{array}{c}
1+0.1 \sin \sqrt{2} t \\
1.2+0.2 \cos \sqrt{3} t
\end{array}\right) \\
& \left(\begin{array}{l}
\eta_{1}(t) \\
\eta_{2}(t)
\end{array}\right)=\left(\begin{array}{c}
0.15+0.02 \sin \sqrt{2} t \\
0.16+0.012 \cos \sqrt{3} t
\end{array}\right) \\
& \left(\begin{array}{ll}
b_{11}(t) & b_{12}(t) \\
b_{21}(t) & b_{22}(t)
\end{array}\right)=\left(\begin{array}{cc}
0.1 e_{0} \sin \sqrt{6} t+0.2 e_{1} \sin \sqrt{6} t & 0.13 e_{0}+0.1 e_{12} \sin \sqrt{7} t \\
0.15 e_{0}+0.1 e_{1} \cos \sqrt{5} t+0.2 e_{12} \cos \sqrt{2} t & 0.11 e_{0}+0.2 e_{2} \sin \sqrt{3} t
\end{array}\right), \\
& \left(\begin{array}{ll}
c_{111}(t) & c_{112}(t) \\
c_{121}(t) & c_{122}(t)
\end{array}\right)=\left(\begin{array}{cc}
0.21 e_{0} \sin \sqrt{6} t+0.12 e_{1} \sin \sqrt{3} t & 0.11 e_{0}+0.12 e_{12} \sin \sqrt{2} t \\
0.14 e_{0}+0.11 e_{1} \cos \sqrt{5} t+0.12 e_{12} \cos 2 \sqrt{5} t & 0.12 e_{0}+0.12 e_{2} \sin \sqrt{3} t
\end{array}\right), \\
& \left(\begin{array}{ll}
c_{211}(t) & c_{212}(t) \\
c_{221}(t) & c_{222}(t)
\end{array}\right)=\left(\begin{array}{cc}
0.11 e_{0} \sin \sqrt{6} t+0.12 e_{1} \sin \sqrt{5} t & 0.11 e_{0}+0.12 e_{12} \sin \sqrt{7} t \\
0.14 e_{0}+0.11 e_{1} \cos \sqrt{5} t+0.12 e_{12} \cos 2 \sqrt{3} t & 0.12 e_{0}+0.12 e_{2} \sin \sqrt{3} t
\end{array}\right), \\
& \left(\begin{array}{ll}
\sigma_{111}(t) & \sigma_{112}(t) \\
\sigma_{121}(t) & \sigma_{122}(t)
\end{array}\right)=\left(\begin{array}{cc}
0.002 \sin \sqrt{6} t+0.01 & 2-\sin t \\
1-0.1 \cos \sqrt{7} t & 0.001 \sin \sqrt{2} t+0.01
\end{array}\right), \\
& \left(\begin{array}{ll}
\sigma_{211}(t) & \sigma_{212}(t) \\
\sigma_{221}(t) & \sigma_{222}(t)
\end{array}\right)=\left(\begin{array}{cc}
0.012 \sin \sqrt{3} t+0.02 & 0.1-0.03 \cos t \\
2-\sin t^{2} & 0.01 \sin \sqrt{6} t+0.011
\end{array}\right), \\
& \left(\begin{array}{ll}
\nu_{111}(t) & v_{112}(t) \\
\nu_{121}(t) & \nu_{122}(t)
\end{array}\right)=\left(\begin{array}{cc}
0.02 \sin \sqrt{5} t+0.03 & 1-0.7 \sin t \\
3-2 \cos \sqrt{2} t & 0.001 \sin \sqrt{7} t+0.01
\end{array}\right), \\
& \left(\begin{array}{ll}
\nu_{211}(t) & \nu_{212}(t) \\
\nu_{221}(t) & \nu_{222}(t)
\end{array}\right)=\left(\begin{array}{cc}
0.002 \sin \sqrt{3} t+0.02 & 5-3 \cos t \\
0.1-0.07 \sin t & 0.01 \sin \sqrt{8} t+0.015
\end{array}\right), \\
& \left(\begin{array}{l}
Q_{1}(t) \\
Q_{2}(t)
\end{array}\right)=\left(\begin{array}{l}
\frac{1}{15} e_{0} \sin 2 \sqrt{5} t+\frac{1}{20} e_{1} \sin \sqrt{3} t+\frac{1}{12} e_{2} \cos \sqrt{3} t+\frac{1}{15} e_{12} \sin \sqrt{7} t \\
\frac{1}{15} e_{0} \sin 2 \sqrt{3} t+\frac{1}{12} e_{1} \sin \sqrt{6} t+\frac{1}{10} e_{2} \cos \sqrt{7} t+\frac{1}{20} e_{12} \sin ^{2} \sqrt{3} t
\end{array}\right) .
\end{aligned}
$$



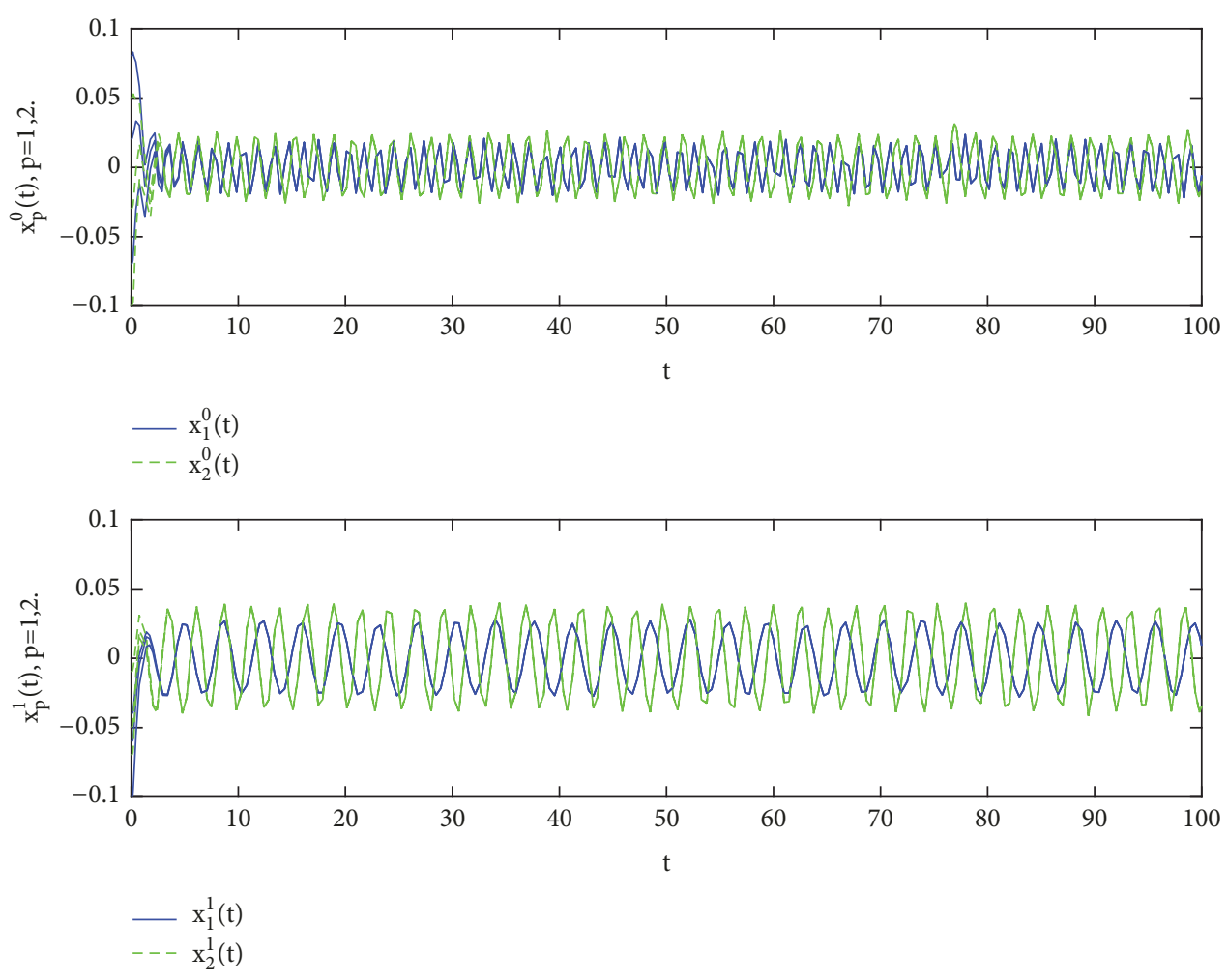

Figure 1: Curves of $x_{p}^{0}(t)$ and $x_{p}^{1}(t), p=1,2$.

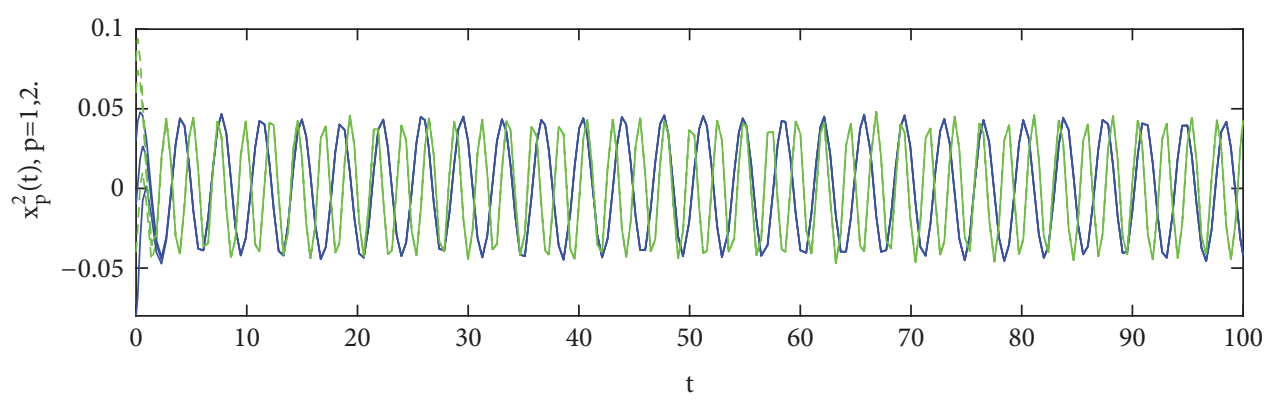

$-x_{1}^{2}(t)$
$---x_{2}^{2}(t)$

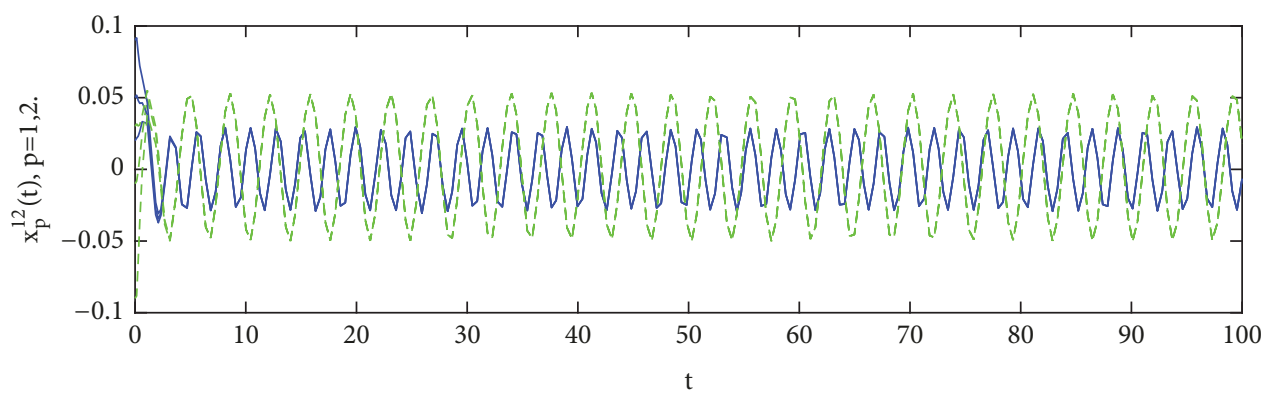

$\mathrm{x}_{1}^{12}(\mathrm{t})$
$--\mathrm{x}_{2}^{12}(\mathrm{t})$

FIGURE 2: Curves of $x_{p}^{2}(t)$ and $x_{p}^{12}(t), p=1,2$. 

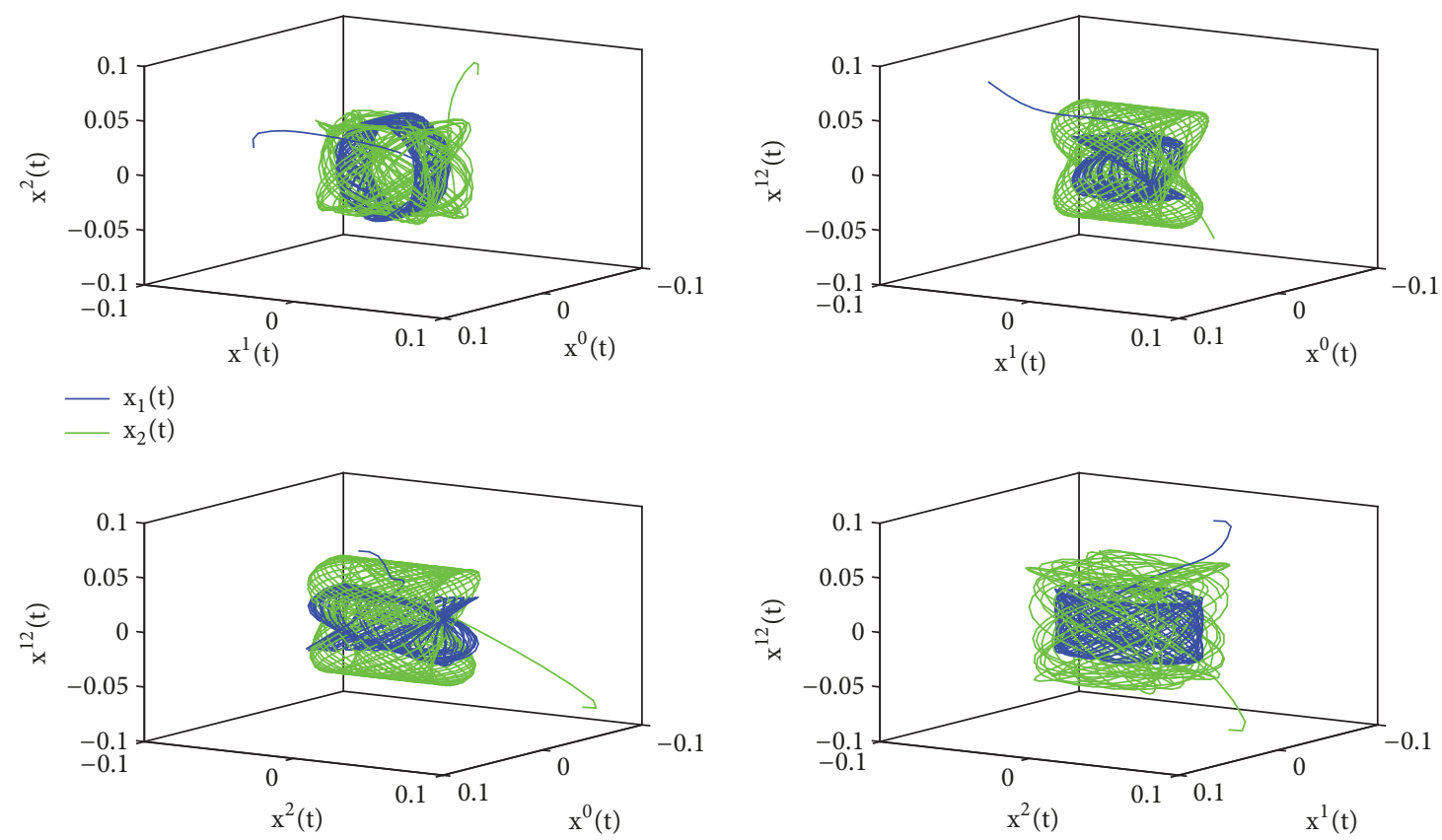

Figure 3: Curves of $x^{0}(t), x^{1}(t), x^{2}(t)$, and $x^{12}(t)$ in 3-dimensional space for stable case.

By computing, we get $L_{q}^{f}=1 / 20, L_{q}^{g}=1 / 15, G_{q}=0.067$, $q=1,2, a_{1}^{-}=0.9, a_{2}^{-}=1, a_{1}^{+}=1.1, a_{2}^{+}=1.4, \eta_{1}^{+}=0.17, \eta_{2}^{+}=$ $0.172, b_{11}^{+}=0.2, b_{12}^{+}=0.13, b_{21}^{+}=0.2, b_{22}^{+}=0.2, c_{111}^{+}=0.21$, $c_{112}^{+}=0.12, c_{121}^{+}=0.14, c_{122}^{+}=0.12, c_{211}^{+}=0.12, c_{212}^{+}=0.12$, $c_{221}^{+}=0.14, c_{222}^{+}=0.12, M_{1}=0.2088, M_{2}=0.2653$, and

$$
r=\max _{1 \leq p \leq 2}\left\{\frac{1}{a_{p}^{-}} M_{p},\left(1+\frac{a_{p}^{+}}{a_{p}^{-}}\right) M_{p}\right\} \approx 0.6366<1 .
$$

Therefore, all of the conditions of Theorem 8 are satisfied. Hence, system (2) has one almost automorphic solution that is globally exponentially stable (see Figures 1-3).

\section{Conclusion}

In this paper, we obtained the existence and global exponential stability of almost automorphic solutions for Cliffordvalued high-order Hopfield neural networks by direct method. Our methods and results are new. The methods proposed in this paper can be used to study the problem of almost automorphic solutions of other types of Cliffordvalued neural networks with or without leakage delays such as Clifford-valued BAM networks, Clifford-valued cellular neural networks, and Clifford-valued shunting inhibitory cellular neural networks. Studying the dynamics of the Cliffordvalued neural networks with impulsive effects is our future work.

\section{Data Availability}

No data were used to support this study.

\section{Conflicts of Interest}

The authors declare that they have no conflicts of interest.

\section{Acknowledgments}

This work is supported by the National Natural Science Foundation of China under Grant No. 11861072.

\section{References}

[1] B. Xu, X. Liu, and X. Liao, "Global asymptotic stability of high-order Hopfield type neural networks with time delays," Computers \& Mathematics with Applications, vol. 45, no. 10-11, pp. 1729-1737, 2003.

[2] X. Liu, K. Teo, and B. Xu, "Exponential stability of impulsive high-order hopfield-type neural networks with time-varying delays," IEEE Transactions on Neural Networks and Learning Systems, vol. 16, no. 6, pp. 1329-1339, 2005.

[3] X.-Y. Lou and B.-T. Cui, "Novel global stability criteria for highorder Hopfield-type neural networks with time-varying delays," Journal of Mathematical Analysis and Applications, vol. 330, no. 1, pp. 144-158, 2007.

[4] H. Xiang, K. M. Yan, and B. Y. Wang, "Existence and global exponential stability of periodic solution for delayed high-order Hopfield-type neural networks," Physics Letters, vol. 352, no. 45, pp. 341-349, 2006.

[5] L. Duan, L. Huang, and Z. Guo, "Stability and almost periodicity for delayed high-order Hopfield neural networks with discontinuous activations," Nonlinear Dynamics, vol. 77, no. 4, pp. 1469-1484, 2014.

[6] C. Aouiti, M. S. M'hamdi, J. Cao, and A. Alsaedi, "Piecewise pseudo almost periodic solution for impulsive generalised highorder hopfield neural networks with leakage delays," Neural Processing Letters, vol. 45, no. 2, pp. 615-648, 2017. 
[7] C. Aouiti, "Oscillation of impulsive neutral delay generalized high-order Hopfield neural networks," Neural Computing and Applications, vol. 29, no. 9, pp. 477-495, 2018.

[8] L. Duan, L. Huang, Z. Guo, and X. Fang, "Periodic attractor for reaction-diffusion high-order Hopfield neural networks with time-varying delays," Computers \& Mathematics with Applications, vol. 73, no. 2, pp. 233-245, 2017.

[9] A. M. Alimi, C. Aouiti, F. Chérif, F. Dridi, and M. S. M'hamdi, "Dynamics and oscillations of generalized high-order Hopfield neural networks with mixed delays," Neurocomputing, vol. 321, pp. 274-295, 2018.

[10] Y. Li, J. Qin, and B. Li, "Anti-periodic solutions for quaternionvalued high-order hopfield neural networks with time-varying delays," Neural Processing Letters, vol. 49, no. 3, pp. 1217-1237, 2019.

[11] Y. K. Li, X. F. Meng, and L. L. Xiong, "Pseudo almost periodic solutions for neutral type high-order Hopfield neural networks with mixed time-varying delays and leakage delays on time scales," International Journal of Machine Learning and Cybernetics, vol. 8, no. 6, pp. 1915-1927, 2017.

[12] C. Aouiti and E. A. Assali, "Stability analysis for a class of impulsive high-order Hopfield neural networks with leakage time-varying delays," Neural Computing and Applications, pp. 1-23, 2018.

[13] L. Zhao, Y. Li, and B. Li, "Weighted pseudo-almost automorphic solutions of high-order Hopfield neural networks with neutral distributed delays," Neural Computing and Applications, vol. 29, no. 7, pp. 513-527, 2018.

[14] N. Huo, B. Li, and Y. Li, "Existence and exponential stability of anti-periodic solutions for inertial quaternion-valued highorder hopfield neural networks with state-dependent delays," IEEE Access, vol. 7, pp. 60010-60019, 2019.

[15] W. K. Clifford, "Applications of grassmann's extensive algebra," American Journal of Mathematics, vol. 1, no. 4, pp. 350-358, 1878.

[16] J. Pearson and D. Bisset, "Neural networks in the Clifford domain," in Proceedings of IEEE World Congress on Computational Intelligence (WCCI), International Conference on Neural Networks (ICNN), vol. 3, pp. 1465-1469, Orlando, FL, USA, 1994.

[17] S. Buchholz and G. Sommer, "On Clifford neurons and Clifford multi-layer perceptrons," Neural Networks, vol. 21, no. 7, pp. 925-935, 2008.

[18] Y. Liu, P. Xu, J. Lu, and J. Liang, "Global stability of Cliffordvalued recurrent neural networks with time delays," Nonlinear Dynamics, vol. 84, no. 2, pp. 767-777, 2016.

[19] J. Zhu and J. Sun, "Global exponential stability of Cliffordvalued recurrent neural networks," Neurocomputing, vol. 173, pp. 685-689, 2016.

[20] Y. Li and J. Xiang, "Existence and global exponential stability of anti-periodic solution for Clifford-valued inertial Cohen-Grossberg neural networks with delays," Neurocomputing, vol. 332, pp. 259-269, 2019.

[21] Y. Li and J. Xiang, "Global asymptotic almost periodic synchronization of clifford-valued cnns with discrete delays," Complexity, vol. 2019, Article ID 6982109, 13 pages, 2019.

[22] Y. Li, J. Xiang, and B. Li, "Globally asymptotic almost automorphic synchronization of clifford-valued RNNs with delays," IEEE Access, vol. 7, pp. 54946-54957, 2019.

[23] C. Huang, Z. Yang, T. Yi, and X. Zou, "On the basins of attraction for a class of delay differential equations with nonmonotone bistable nonlinearities," Journal of Differential Equations, vol. 256, no. 7, pp. 2101-2114, 2014.
[24] L. Duan, H. Wei, and L. Huang, "Finite-time synchronization of delayed fuzzy cellular neural networks with discontinuous activations," Fuzzy Sets and Systems, vol. 361, pp. 56-70, 2019.

[25] K. Gopalsamy, "Leakage delays in BAM," Journal of Mathematical Analysis and Applications, vol. 325, no. 2, pp. 1117-1132, 2007.

[26] Y. Li and X. Meng, "Existence and global exponential stability of pseudo almost periodic solutions for neutral type quaternionvalued neural networks with delays in the leakage term on time scales," Complexity, vol. 2017, Article ID 9878369, 15 pages, 2017.

[27] F. Brackx, R. Delanghe, and F. Sommen, Clifford Analysis, vol. 76 of Research Notes in Mathematics, Pitman, Advanced Publishing Program, Boston, MA, USA, 1982.

[28] T. Diagana, Almost Automorphic Type and Almost Periodic Type Functions in Abstract Spaces, Springer, New York, NY, USA, 2013. 


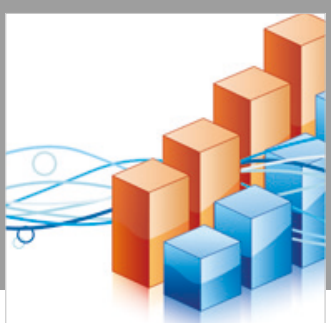

Advances in

Operations Research

\section{-n-m}
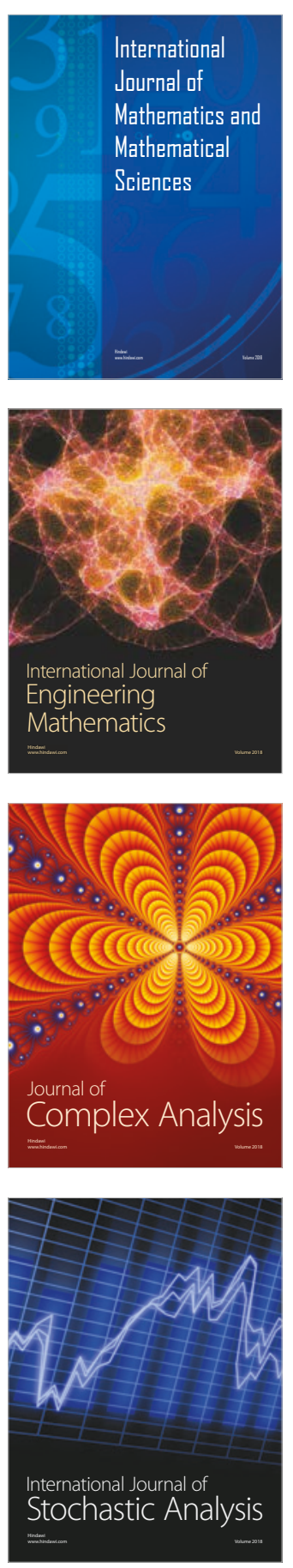
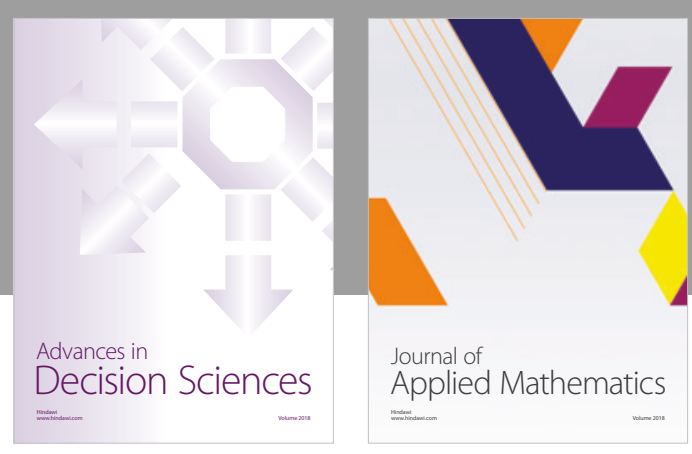

Journal of

Applied Mathematics
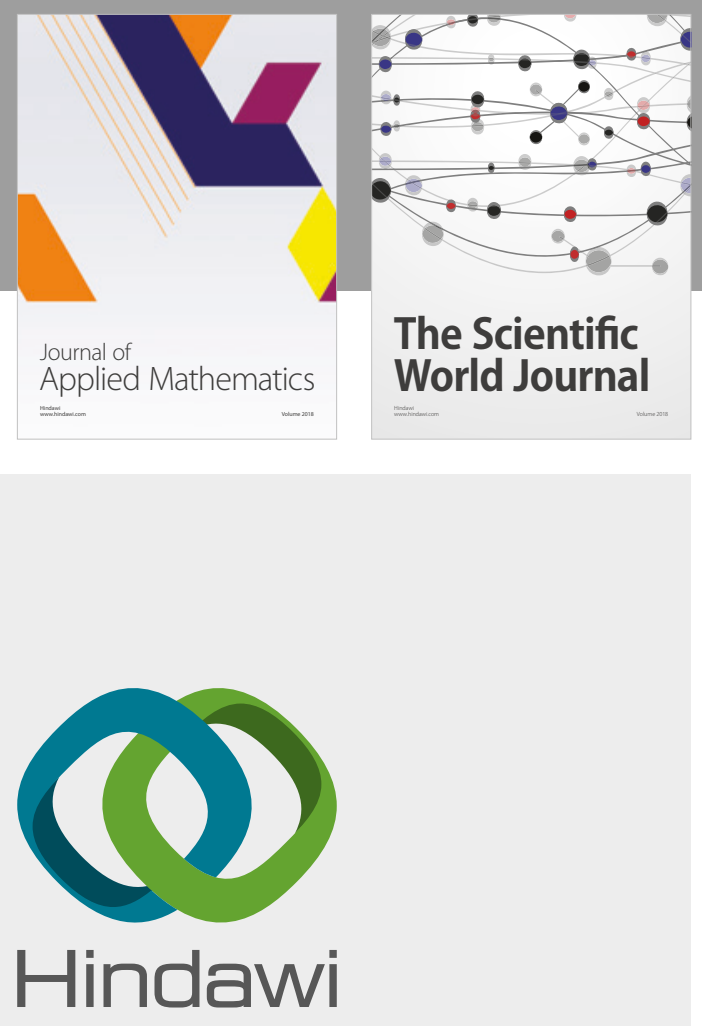

Submit your manuscripts at

www.hindawi.com

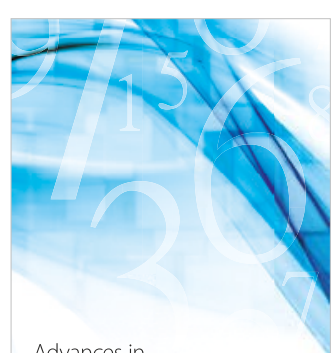

Advances in
Numerical Analysis
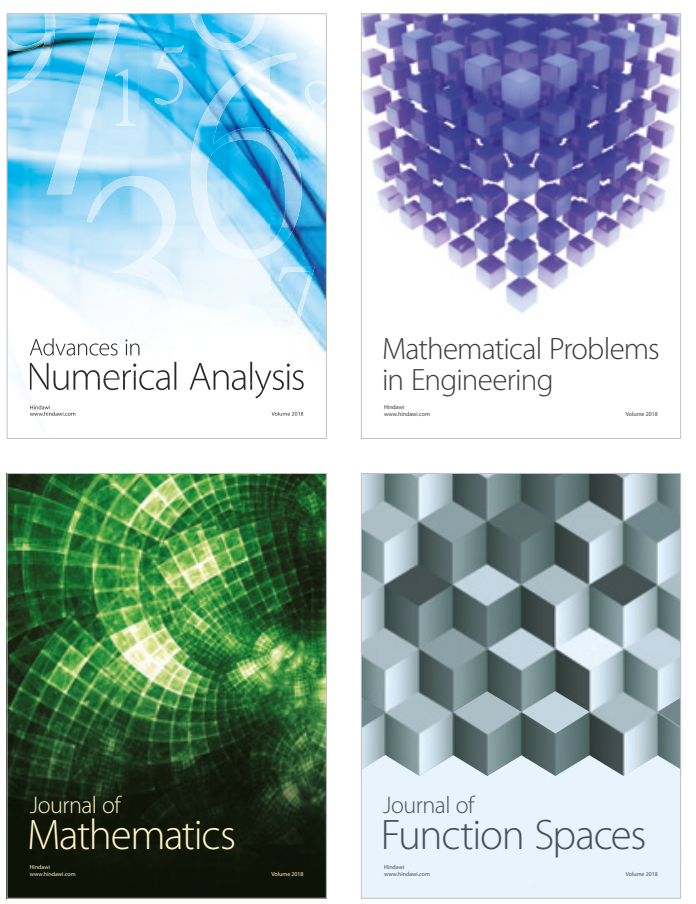

Mathematical Problems in Engineering

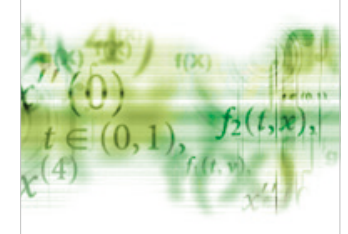

International Journal of

Differential Equations

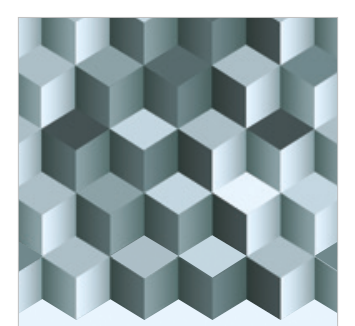

Journal of

Function Spaces
The Scientific

World Journal

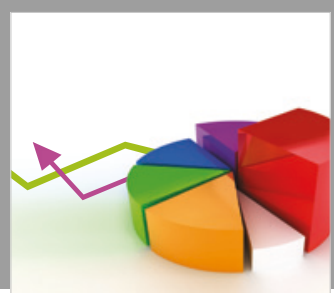

Journal of

Probability and Statistics
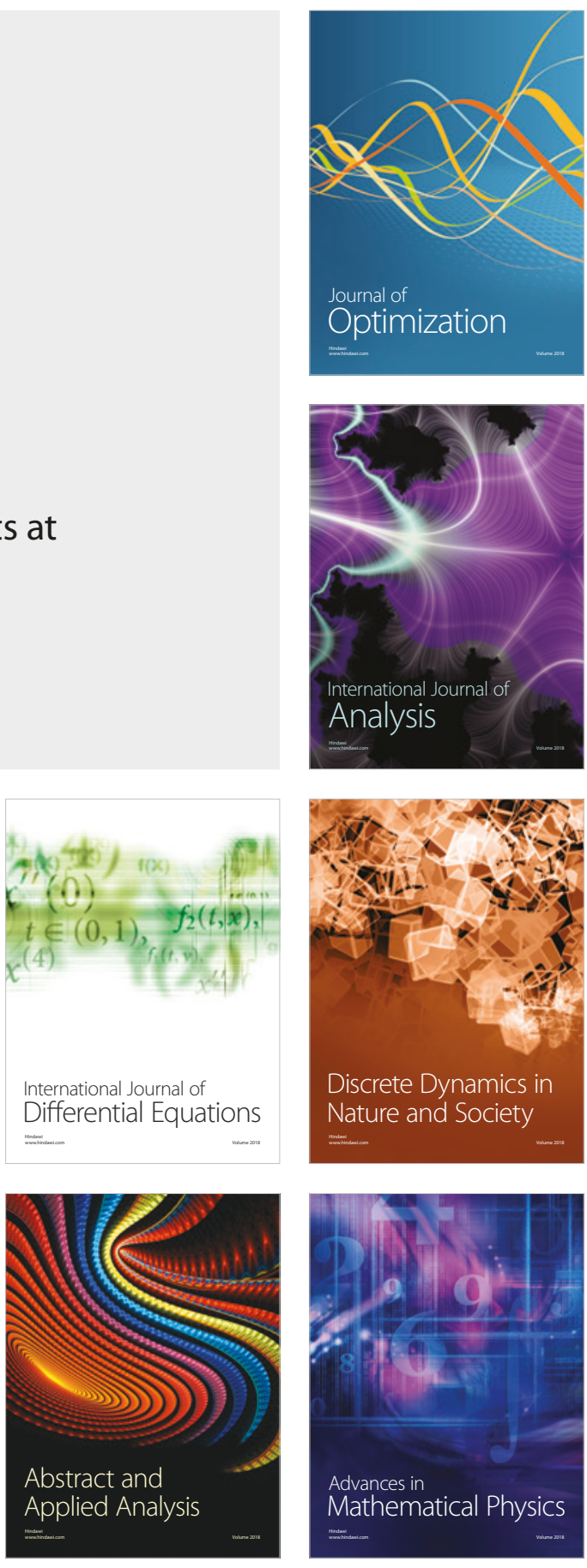